Zbl Arbeitsmed 2015 - 65:125-126

DOI 10.1007/s40664-015-0028-x

Online publiziert: 18. Juni 2015

(c) Springer-Verlag Berlin Heidelberg 2015

CrossMark
D.A. Groneberg ${ }^{1} \cdot$ M. Jäger ${ }^{2} \cdot$ M. Kretz ${ }^{3}$

${ }^{1}$ Institut für Arbeitsmedizin, Sozialmedizin und Umweltmedizin, Goethe-Universität Frankfurt am Main, Frankfurt am Main, Deutschland

${ }^{2}$ Leibniz-Institut für Arbeitsforschung an der TU Dortmund, Dortmund, Deutschland

${ }^{3}$ Springer-Verlag, Heidelberg, Deutschland

\title{
Das Zentralblatt wünscht Professor Luttmann Glück und Gesundheit zu seinem Ruhestand als Hauptschriftleiter
}

Editorials dieser Art kommen im Zentralblatt für Arbeitsmedizin, Arbeitsschutz und Ergonomie nur in Abständen von Jahrzehnten vor und verdienen deswegen ganz besondere Aufmerksamkeit: In diesem Jahr verabschiedet sich Professor Dr. Alwin Luttmann (• Abb. 1) von seinem Amt als einer der beiden federführenden Hauptschriftleiter des Zentralblatts.

Professor Luttmann dient der deutschen Wissenschaft in dieser Funktion über fast 15 Jahre, und der Verlag und das Herausgebergremium möchten ihn deswegen für seine Verdienste ganz besonders ehren. Seit 2001 prägte er an dieser prominenten Stelle die Geschicke des Zentralblatts, zuerst gemeinsam mit Professor Gustav Schäcke, dann ab 2007 gemeinsam mit Professor David Groneberg, wobei er vor allem für die Belange der Ergonomie und des Arbeitsschutzes verantwortlich zeichnete - unter dem Kürzel Hauptschriftleiter Technik.

\section{》) Seit 2001 prägte \\ Professor Luttmann die Geschicke des Zentralblatts}

Bei seiner Berufung in das Gremium hatte er bereits eine große Berufserfahrung aufzuweisen und langjährige Leitungsfunktionen in der Abteilung Ergonomie des Instituts für Arbeitsphysiologie an der Universität Dortmund inne. Sein Interesse für diesen brückenbildenden Bereich zwischen der grundlagenorientierten Physiologie und deren Anwendung in einer arbeitsphysiologisch begründeten Ergonomie wurde bereits früh deutlich: Alwin Luttmann studierte zwischen 1963 und 1969 Elektrotechnik an der Technischen Universität Hannover. Nach etwa 1-jähriger Tätigkeit als dynamisch junger Diplomingenieur in der Sicherheitstechnik an der Physikalisch-Technischen Bundesanstalt in Braunschweig wechselte er sein Tätigkeitsfeld grundlegend. Wissensdurstig und tatenhungrig wurde er wissenschaftlicher Mitarbeiter im Institut für Physiologie an der Ruhr-Universität Bochum (RUB) und legte damit den Grundstein zu einer erfolgreichen wissenschaftlichen Laufbahn. Dem brückenbildenden Gedanken folgend, promovierte Dipl.-Ing. Alwin Luttmann in der als weltoffen geltenden Abteilung für Biologie der noch jungen RUB, habilitierte schon 1980 in der Abteilung für Naturwissenschaftliche Medizin und erhielt als elektrotechnisch ausgebildeter, signaltheoretisch gebildeter und humanfunktionsgefesselter Privatdozent die Venia Legendi für das Fach Physiologie.

Trotz seines Wechsels zum seinerzeitigen Institut für Arbeitsphysiologie an der Universität Dortmund (IfADo) blieb Priv.-Doz. Dr. rer. nat. Dipl.-Ing. Alwin Luttmann „seiner Ruhr-Universität“ für eine gewisse Zeit einer Lehrstuhlvertretung der Physiologie erhalten; darüber hinaus hat er bis in die jüngere Vergangenheit entsprechende Vorlesungen mit ausgeprägtem innerem Engagement sowie mit großer Freude für sich und „seine Studenten“ in Bochum und Dortmund gehalten. Im Jahr 1987 wurde er dann von der Medizinischen Fakultät zum außerplanmäßigen Professor ernannt. In der Abteilung Ergonomie am IfADo übernahm er umgehend inhaltliche und organisatorische Leitungsfunktionen und widmete sich vor allem der Ermittlung muskulärer Beanspruchungen und skeletaler Belastungen in systematischen Laboruntersuchungen und in von den Unvorhersehbarkeiten des Arbeitsalltags geprägten Feldstudien - Letztere lagen ihm besonders am Herzen. Die erforderliche Modernisierung des IfADo Mitte der 1990er-Jahre von einer direktoralen Abteilungs- zu einer dynamischen Projektstruktur war von seinen Ideen wesentlich mitgeprägt, und so muss die Leitungsübernahme einer sehr aktiven Projektgruppe zur Arbeitsphysiologischen Analyse muskuloskeletaler Belastungen geradezu als natürliche Folge erscheinen. Als besonderes Merkmal seines Wirkens ist dabei hervorzuheben, dass er auch stets bereit war, die Angemessenheit von Methoden und Verfahren zu hinterfragen, um letztendlich die Qualität der Ergebnisse und Ermittlungswerkzeuge sowie den Wissenstand zu erhöhen. Er folgte somit dem Ziel, Arbeitsgestaltungsmaßnahmen sowohl im Einzelfall als auch auf Prinzipebene eher evidenz- als eminenzbasiert ableiten und empfehlen zu wollen.

Der wissenschaftliche „Ertrag“ der Arbeit von Alwin Luttmann wird unter anderem an den annähernd 500 Publikationen deutlich, die in nationalen und - schon von Anbeginn an - auch vielfach in inter- 


\section{Editorial}

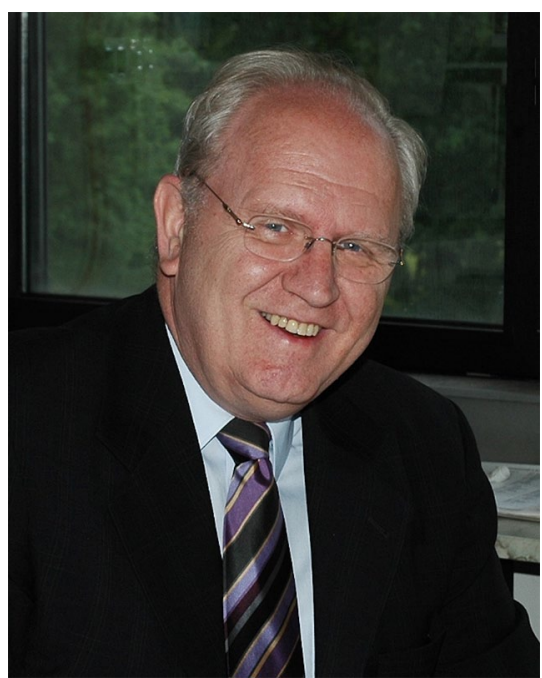

Abb. $1 \Delta$ Prof. Dr. Alwin Luttmann

nationalen Zeitschriften erschienen sind. Sein entwicklungsfreudiges Arbeiten hat auch dazu geführt, dass mehrere Verfahren und Instrumentarien patentiert wurden. Er erhielt zudem eine Reihe von Auszeichnungen und Preisen, wie z. B. die nach seinem Leitbild benannte JosephRutenfranz-Medaille für seine besonderen Verdienste um die Arbeitsphysiologie oder auch den Evamaria-Kinne-Saffran-Preis für eine erfolgreiche Zusammenarbeit verschiedener biomedizinischer Forschungsgruppen. Die Vielfalt seines Erfolges drückt sich darüber hinaus in der Anerkennung seines Sachverstands aus, sei es als ungezählt eingeladener Redner zu Kongressen, als Erläuterer in Fortund Weiterbildungsveranstaltungen oder auch als Gutachter in juristischen Streitfällen arbeitswissenschaftlicher und arbeitsmedizinischer Sachverhalte.

Sein erfolgreiches Wirken und menschlich vorbildliches Verhalten war es auch, das den aktuellen federführenden Hauptschriftleiter Medizin dazu bewog, im Jahr 2007 als Nachfolger des emeritierten, langjährigen medizinischen Hauptschriftleiters Gustav Schäcke in die Schriftleitung einzutreten, um gemeinsam mit Alwin Luttmann die damals durchaus schwierige Lage des Zentralblatts zu überwinden. In gegenseitigem Vertrauen und respektvoller Zusammenarbeit wurde dies auch mithilfe eines verjüngten und vergrößerten Gremiums in der Schriftleitung geschafft.
Zuerst konnte die Attraktivität des Zentralblatts als erstrebenswertem Publikationsmedium gesteigert und vor Kurzem wieder seinem Ursprungsverlag, dem Springer-Verlag, zugeführt werden. Das positive Echo der Leserschaft und die ehrenvollen Geleitwörter von Bundes- und Staatsministern zu verschiedenen Stationen des Zentralblatts zeigen, dass Alwin Luttmann seine Energie in ein ganz wichtiges Unterfangen stellte - nämlich der Publikation aktueller arbeitswissenschaftlicher und arbeitsmedizinischer Kenntnisse.

\section{》) Professor Luttmann}

steckte viel Energie in die Publikation aktueller arbeitswissenschaftlicher und arbeitsmedizinischer Kenntnisse

Seit Anbeginn seiner Arbeiten - nicht nur im Zentralblatt - bedeutete es Alwin Luttmann eine große Freude, sein Wissen und das Wissen anderer an Interessierte weiterzugeben, sowohl im Rahmen von Vorlesungen an den Universitäten in Bochum, Dortmund und Zürich als auch im Rahmen von mehreren hundert Beiträgen auf wissenschaftlichen Tagungen und nicht zuletzt in seiner Tätigkeit als die Feder führender Schriftleiter. Seine diesbezügliche Bilanz ist beeindruckend: Seit dem 01.01.2001 zeichnet er verantwortlich für die Herausgabe von über 500 wissenschaftlichen Originalarbeiten im Zentralblatt; diese wurden in über 150 einzelnen Heften verlegt. Durch sein nimmermüdes und beharrliches Wirken hat sich Herr Luttmann einen großen Verdienst am Fortbestand des vorliegenden Publikationsorgans erworben.

Dabei verdeutlicht die stets verständnisvolle Art im Umgang mit Autoren verschiedenster Fachbereiche, die sich alle im interdisziplinären Schmelzpunkt von Arbeitswissenschaften, Arbeitsmedizin und Arbeitsschutz wiederfinden, einen wesentlichen Charakterzug des Menschen Alwin Luttmann. Dieser Wesensart ist es auch zu verdanken, dass das Zentralblatt bis heute von allen relevanten Interessengruppen als ein neutrales und objektives Publikationsorgan geachtet wird, das alle Mehrheits- und Minderheitsmei- nungen auf der Basis gesicherter wissenschaftlicher Grundlagen zu Wort kommen lässt. Dieser Rahmen führt seinen aktuellen und künftigen Nachfolgern in der Leitung einer der ältesten deutschen Fachzeitschriften des Gebiets klare Leitlinien vor Augen.

Verlag und Schriftleitung des Zentralblatts danken dem scheidenden Hauptschriftleiter mehr als herzlich für die stets angenehme und fruchtvolle gemeinsame Zeit und wünschen Herrn Prof. Luttmann alles Gute sowie Glück, Gesundheit und Zufriedenheit für die kommenden Jahre und viel Spaß bei der Lektüre seines Zentralblatts.

Prof. Dr. David A. Groneberg

Federführender Hauptschriftleiter

Medizin

PD Dr. Matthias Jäger

Federführender Hauptschriftleiter

Technik

Monika Kretz

Head of Journals \& ePublishing

\section{Korrespondenzadresse}

Prof. Dr. D.A. Groneberg

Institut für Arbeitsmedizin, Sozialmedizin und Umweltmedizin

Goethe-Universität Frankfurt am Main

60590 Frankfurt am Main

arbsozmed@uni-frankfurt.de

Interessenkonflikt. D.A. Groneberg, M. Jäger und M. Kretz geben an, dass kein Interessenkonflikt besteht. 